\title{
Achieving a Responsive Philippine Health Research Agenda: An Analysis of Research Outputs and Underlying Factors
}

\author{
Jaifred Christian F. Lopez, Teddy S. Dizon and Regin George Miguel K. Regis \\ Alliance for Improving Health Outcomes
}

\begin{abstract}
Introduction. Priority setting in the context of health research is important for developing countries as it assists policymakers and researchers in effectively and efficiently targeting research topics with the greatest benefit for public health. In the local setting, the Philippine National Unified Health Research Agenda (NUHRA) was formulated to prioritize health research topics that address the health needs of the population alongside the health sector's goal for universal health care. This paper attempts to understand the alignment of the health research outputs by the Philippine Council for Health Research and Development (PCHRD) in addressing the priority health needs of the country.
\end{abstract}

Methods. Review of secondary data and key informant interviews was done. Using a thematic analysis approach, we extracted secondary data from the 2006 to 2017 PCHRD Annual Reports to identify the completed PCHRDfunded health research projects. Excluded in scoping of secondary data were the zonal and other unpublished or not readily available online reports.

Results. Results show that most research outputs address NUHRA priorities on health technology development and on the health needs on all life stages and triple burden of diseases under the Philippine Health Agenda. However, for research outputs to improve health outcomes, efforts must first be focused on health systems strengthening and the utilization of existing research information.

Conclusion. Gaps and challenges in the previous NUHRAs suggest that even as researches are aligned to the agenda, they do not necessarily address the health needs of the Philippines.

Key Words: support of research, health research, health priorities

\section{INTRODUCTION}

Health research capacity, or the improvement of human and institutional resources to better conduct, analyze and utilize health research, is increasingly recognized as a sustainable strategy for overcoming public health challenges, especially for developing countries. ${ }^{1}$ In its 1990 report, the Commission on Health Research for Development emphasized that strengthening research capacity in developing countries is "one of the most powerful, costeffective and sustainable means of advancing health and development." ${ }^{1}$ By improving the capacity to effectively and

Corresponding author: Teddy S. Dizon, RN, DIH Alliance for Improving Health Outcomes 62 West Avenue, Quezon City 1104, Philippines Telephone: +639173082178

Email: tsdizon1@up.edu.ph efficiently define problems and set objectives and priorities, and build sustainable institutions and organizations using knowledge gathered from health research, health systems in developing countries are equipped with the necessary data that can help resolve key national health issues. ${ }^{2}$ 
Nonetheless, this potential is hampered by insufficient financial resources and facilities to support health research. ${ }^{3}$ As such, researchers in low- and middle-income countries (LMICs) have been constrained to focus efforts on generating research that targets identified needs and priorities of the country. This approach has evolved into the development of a national health research agenda. In the Philippines, the National Unified Health Research Agenda (NUHRA) sets the research priorities determined by the country's stakeholders and addresses the health needs of the population vis-a-vis the health sector's goal for universal health care. ${ }^{4}$ It aims to develop knowledge to understand health challenges and to mount an improved response to them, ${ }^{4}$ while also aiming to ensure that health research is responsive to the health systems need and that investments yield the most benefit. ${ }^{5}$ The process of developing health research agenda has been implemented thrice starting with its inaugural version in 2006, with each version covering a five-year period henceforth. Notably, the development of these research agenda involved various stakeholders and employed different strategies. Specifically, the 20062011 version (NUHRA 1) implemented a consultative process that focused on health needs sourced from key players directly involved with health systems and research, while the 2012-2017 version (NUHRA 2) started with programmatic thrusts of the key agencies involved in the Philippine National Health Research System (PNHRS), and thereafter gathered inputs from stakeholders. The current version, the 2017-2022 version sought to achieve the middle ground between the two strategies utilized in the preceding versions. ${ }^{5}$

While each version has its unique approach and limitations, it cannot be denied that the development of health research agenda facilitated inter-agency cooperation, strengthened support and camaraderie among researchers, and highlighted the importance of research in improving healthcare. However, an important question remains to be answered if a research agenda is indeed responsive to the health needs of the Philippines. In proposing this question, we consider responsiveness from a systems perspective, in that it is defined as how well the health systems in place meets the "legitimate expectations" of the population. ${ }^{6}$ Therefore, in the context of health research agenda setting, we consider health research agenda as responsive if the research outputs address health needs, under the assumption that the health systems should address the fundamental needs or the basic human rights of Filipinos. ${ }^{6}$ Thus, guided by these definitions, we attempted to analyze if outputs of research that were aligned closely with priority topics of past versions of the NUHRA indeed endeavored to address the priority health needs of the Philippines.

This approach is influenced by the assumption that research grant applications submitted to the Philippine Council for Health Research and Development (PCHRD), the foremost funding agency for health research under the
Department of Science and Technology (DOST), should be aligned to the NUHRA and approval of each grant proposal was considered a confirmation of its alignment with the research agenda.

Meanwhile, we defined "health needs" as those which are listed in the Philippine Health Agenda, a health reform framework which includes health priorities from the three past Philippine presidential administrations.

\section{MATERIALS AND METHODS}

Using a thematic analysis approach, we extracted secondary data from the 2006 to 2017 PCHRD Annual Reports to identify the completed PCHRD-funded health research projects. ${ }^{7-18}$ Excluded in scoping of secondary data were the zonal and other unpublished or not readily available online reports. Those that were completed from 2006 to 2011 were considered as NUHRA 1 outputs while those that were completed from 2012-2017 were outputs of NUHRA $2 .{ }^{19,20}$ These data were then categorized according to the thematic priority areas of their respective NUHRA; namely, health financing, governance, health regulations, health service delivery, health technology development, health research ethics, and health information systems for NUHRA 1 and; health technology development, health financing, health service delivery, and socio-environmental health concerns for NUHRA 2. Similarly, the extracted data from both agenda were separately categorized according to the three guarantees in addressing the health needs of the Filipinos indicated in the Philippine Health Agenda 2016-2022 which are: (1) all life stages and triple burden of diseases, (2) service delivery network, and (3) universal health insurance. ${ }^{21}$

Furthermore, five in-depth interviews using an interview guide were conducted among members of the four (4) PNHRS core agencies to aid in contextualizing the responsiveness of the NUHRAs. These four agencies were the DOST-PCHRD, the Department of Health (DOH), the Commission on Higher Education (CHED), and the National Institutes of Health (NIH) based in the University of the Philippines (UP) Manila. The verbal data were recorded and transcribed. To analyze the textual data, the researchers utilized an inductive approach to (a) condense raw textual data into a brief, summary format; (b) establish clear links between the evaluation or research objectives and the summary findings derived from the raw data; and (c) develop a framework of the underlying structure of experiences or processes that are evident in the raw data. ${ }^{21}$

\section{RESULTS AND FINDINGS}

A total of 69 and 160 PCHRD-funded health researches from the 2006-2011 and 2012-2017 NUHRA versions, respectively, were extracted from the PCHRD annual reports. ${ }^{7-18}$ 
Most health researches conducted in the last 10 years addressed knowledge gaps on health technology development, with 39\% in NUHRA 1 and 43\% in NUHRA 2 (Figures 1 and 2). Meanwhile, there were little or no health researches on health systems strengthening in either of the 2 health agendas (Figures 1 and 2).

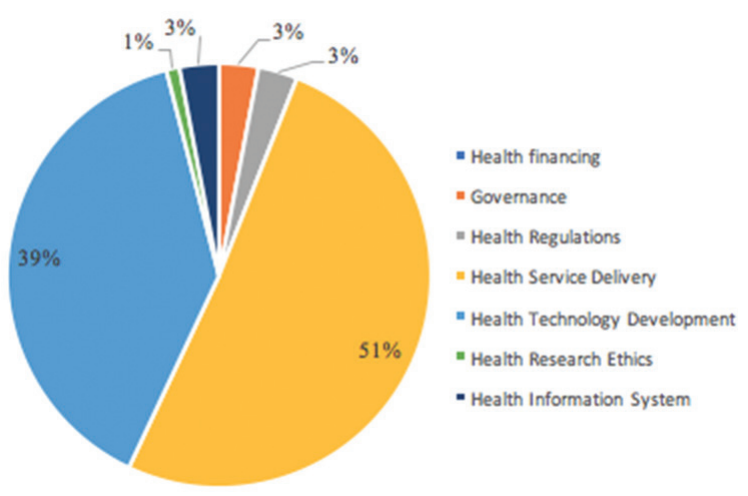

Figure 1. Distribution of health research outputs conducted under NUHRA 1 (2006-2011).

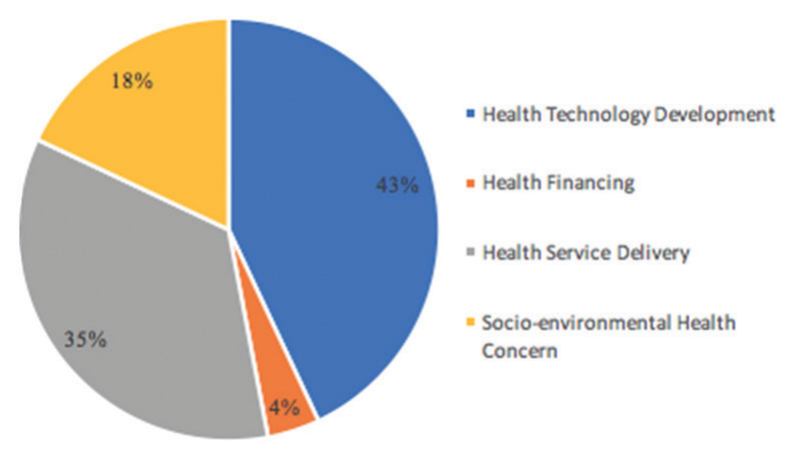

Figure 2. Distribution of health research outputs conducted under NUHRA2 (2012-2017).

Figure 3 shows the distribution of the NUHRA health researches and the corresponding PHA objective that they address. ${ }^{21}$ Most of the completed NUHRA health researches attempted to fill the knowledge gap and develop technologies related to all life stages and the triple burden

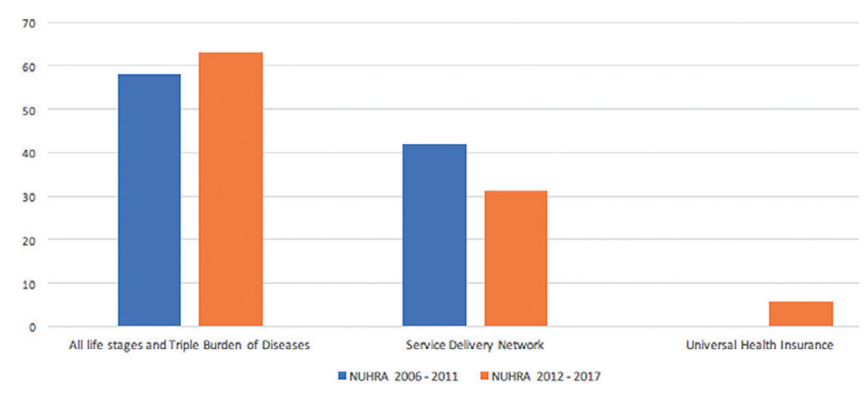

Figure 3. Percentage of NUHRA health research outputs towards addressing the Philippine Health Agenda. of diseases such as genomic and immunology studies; antiviral, antibiotic, and anticancer drug development; vector mapping and prevalence studies on infectious diseases such as dengue, tuberculosis, and leptospirosis; and studies on herbal medicines (e.g. virgin coconut oil, cashew nut extract, mahogany leaves, guava leaves, chinese hibiscus, calabash). There were also several health researches that addressed the service delivery network key programmatic gaps and challenges by evaluating health program planning, implementation, and monitoring. However, there were only a few funded and completed health researches that focused on the challenges faced in implementing and evaluating financial risk protection strategy to achieve better health outcomes.

\section{DISCUSSION}

The primary purpose of this paper was to analyze the responsiveness of the outputs of PCHRD-funded health research projects to the health needs of the Philippines, that is, as emphasized by various studies, should be identified based on epidemiological data and local significance. ${ }^{22-24}$ In its effort to address the health needs of Filipinos, the Philippine government formulated its flagship health plan, the Philippine Health Agenda 2016-2022, which guarantees to address all life stages and triple burden of diseases, service delivery network, and universal health insurance. However, this agenda does not determine the direction of the health researches being conducted in the country. In pursuant to Republic Act 10532, the Philippine National Health Research System (PNHRS) is mandated to formulate the NUHRAs, which have been defined as a set of health research priorities aimed at addressing the national health needs, as determined by the country's stakeholders.

As such, it is not surprising that much emphasis of PCHRD-funded health researches addressed health and technology development the most, followed by health service delivery. Key informants from PCHRD affirmed this finding and added that this was as expected given that "it [health and technology development] is our institutional goal as an agency devoted to developing and strengthening capacity for health science and technology". On the other hand, areas on regulatory, governance and ethics were barely addressed because "these were not, at the time, mainstream and thus, lacked significant research proposals".

However, for countries that are yet to achieve better health outcomes, efforts must be concentrated on conducting contextually relevant researches to inform health systems improvements. ${ }^{25}$ Strengthening health research capacity helps a country address its health needs by establishing a foundation for a well-functioning national health system thereby improving its overall health outcomes. ${ }^{26}$ Three areas must be considered in building capacity for health research: (1) improving the ability to conduct and create demand for research; (2) active participation among institutions, and (3) 
increasing research uptake and utilization. ${ }^{27,28,29}$ However, a number of gaps and challenges in these areas were observed in the implementation of the past NUHRAs.

Conducting innovative and effective research depends on the number and quality of researchers and the facilities they work with. ${ }^{1}$ According to the United Nations Educational, Scientific and Cultural Organization (UNESCO) Institute for Statistics the number of researchers in research and development (that is, "professionals engaged in the creation of new knowledge, products, processes, methods, or systems or in the management of the projects concerned") in the Philippines were 78.2 per million people in 2011 and 189.4 in 2017, which were both less than half of the suggested 380 per million population. ${ }^{30,31}$

Ramos-Jimenez and colleagues reported that the low uptake of NUHRA can be associated with the inadequacy of skilled and quality health researchers in the country, especially in the regions. ${ }^{5,32}$ Additionally, some informants articulated the need to further develop the capacity of regional-based researchers in writing proposals and in implementing health researches and, as well as to strengthen regional and provincial institutions as suggested by the fact that majority of funded NUHRA researches are NCRbased. Studies on capacitating health research in developing countries through human capital have shown that national and institutional programs focusing on providing appropriate trainings, workshops, and seminars and on increasing scholarships lead to increased quality proposals and researches and improved results communication. ${ }^{33,34}$ Currently, PCHRD focuses its efforts on training regional researchers through a workshop called Call for Proposals wherein participants are trained on basic research methods, data processing and analysis, report writing, and utilization of research reports. ${ }^{5}$

However, while skills improvement through training contribute to better research proposal formulation and project implementation, there is a need to sustain the demand for health research. ${ }^{34}$ Majority of the key informants argued that the low NUHRA uptake cannot be predominantly blamed on the capacity of researchers but rather on poor information dissemination of the research agenda itself. The prevailing issue of poor dissemination in NUHRA 1 persisted in NUHRA 2, though with less intensity. The first agenda was regarded with insufficient and ineffective methods of dissemination (workshops and forums designed for introducing NUHRA and various media outlets such as newspapers, radios, and magazines); several sectors were unaware or had limited understanding of the priorities indicated in the NUHRA. Similarly, NUHRA 2 was not well disseminated as there were no sustained national public dissemination or advocacy dedicated to emphasizing the priorities as national health needs; dissemination was limited to the PCHRD anniversary in 2012 and to other conferences hosted by the core agencies where printed copies of the agenda were distributed. As such, some key informants argued that this contributed to low research uptake given that there were a lot of researchers who were unaware of the research priorities.

Kirigia and colleagues emphasize the importance of embedding the identification of an effective dissemination of priorities in the overall health research agenda formulation process. ${ }^{35}$ To create demand for the implemented health research agenda, core agencies must exhaust possible channels in disseminating the health needs to the target audience and in making the processes more explicit. Furthermore, the core agencies must foster the involvement of all parties, including researchers, policymakers and other high government officials, stakeholders both public and private, government services, media, industry, and communities. ${ }^{36}$ Possible channels may include advocacy events relating to health and research, briefings and testimonies to political bodies (e.g., Philippine Senate), meetings and conferences at both the international and national levels, national program reviews, and the technical media..$^{37,38}$

Active participation in the process of formulating a national agenda requires that stakeholders be involved throughout the duration of the process. According to literature review and the key informants interviewed, the core agencies applied a bottom-up and top-down approach at the "regional, zonal and national levels" in gathering inputs through consultations, categorizing them into research priorities "derived from health systems operational frameworks and broad disease categories" ${ }^{39}$ Specifically, the 2006-2011 NUHRA clustered its gathered priorities from nationwide consultation according to the WHO Health Systems building blocks which are service delivery, health workforce, health information systems, health technology, health financing, and governance. ${ }^{40} \mathrm{On}$ the other hand, the 2012-2017 NUHRA focused on the priorities of the four core agencies which are health technology development, health service delivery, health financing, and socioenvironmental health concerns.

Ramos-Jimenez et al.'s assessment on the previous NUHRAs showed that stakeholder participation was an integral component in the formulation of both health research agendas, and rightly so. The inclusion of a "larger and more diverse group of stakeholders" and their active engagement in the whole formulation process yields an agenda that best represents the "system of values of the broader community" ultimately, leading to legitimate recommendations. ${ }^{41,42}$ Thus, the omission of important stakeholders' casts doubt in the "legitimacy of the entire process". ${ }^{39,42}$

Interestingly, Ramos-Jimenez at al. and Tomlinson et al. reported that the formulation process of the NUHRAs were characterized by poor stakeholder involvement and that "not all participants considered the process as relevant". For instance, it was reported, and was confirmed by the key informants from the 4 core agencies, that the 2006-2012 NUHRA having had followed the bottom-up approach, focused on the interests of both the public sector and 
stakeholders, resulting to minimal engagement with the private sector, academe, industry, and other organizations involved in health service delivery. ${ }^{39}$

On the contrary, the top-down approach used to formulate the 2012-2017 NUHRA resulted to an agenda that is "too oriented to the core agencies" thus "unable to capture the needs from down the ground", as mentioned by the key informants. ${ }^{32}$ Consequently, this led Ramos-Jimenez et al. to assess the previous agendas as having "no overarching framework" and "no achievable and measurable indicators". 5,32

Apart from these concerns, a research agenda that deals with indefinite topics necessitates a "revision and appeals" process that paves the way for modifications as based on emerging issues or arguments. ${ }^{42}$ This allows for a means to validate the set health research priorities with the health needs of the country. ${ }^{39}$ However, neither NUHRA has a regular monitoring and evaluation process among stakeholders, and was limited to the assessment of the research uptake after the implementation of the health research agenda.

Among the cited limitations of the previous NUHRAs is low research utilization, that is, the use of research information generated to influence decision-making concerning "policy, advocacy and resource allocation, planning and management, and program systems development and strengthening". ${ }^{43}$ Of the NUHRA 1 completed research outputs, PCHRD reported to only have one that influenced policy and another which contributed to program implementation. Meanwhile, no data regarding research utilization was available for the second NUHRA.

Goodman describes health technology development in three ways: 1) its physical nature, which is the actualization of innovations such as drugs, biologicals, medical devices or instrumentation, and any other mechanical devices involved in information systems, 2) its health care purpose or application such as prevention, screening, diagnosis, and treatment of diseases, and 3) the stage of diffusion whether it be in the conceptual, experimental, investigational, or established stage. ${ }^{44}$ Despite reports of increased uptake of the research priorities on health and technology development, hardly any researches are materialized. This posits barriers in improving health outcomes as researches on health and technology development require practical application to improve or maintain individual and population health. ${ }^{44}$ A key informant expressed the same concern that "the NUHRA is producing researches without proper utilization, resulting in an abundance of unused information." Thus, health technology development researches conducted have purely become theoretical rather than focusing on practical application thus, are not truly helpful in achieving better health outcomes.

We argue in another publication that local institutional readiness remains an essential concern for research utilization to truly respond to the country's health needs, and the results of this analysis help bolster this assertion..$^{39}$ Supporting this further, Almeida and Bascolo and Moore et al. enumerate these challenges in research utilization: 1) interactions between policymakers, researchers, research users and other stakeholders, which include conflicts in political ideology, professional separation between researchers and practitioners and "scientific divergences among researchers; 2) the nature of the evidence and the different contexts of research users, for instance, there exist varying interpretations of risk, in the individual or collective level, on "noxious exposures and disease prevalence"; 3) social constraints such as "media interference" and barriers in research marketing and circulation, and; 4) different timeframes of research and decision-making processes. ${ }^{44,45}$

Supporting these cited challenges, Solidum conducted a study on research utilization in two local government hospitals and pointed out two challenges: 1) the organization subscale and 2) the communication subscale. The former includes issues at the organizational level such as physical infrastructure, lack of political support for staff, and the time allotment in the implementation of research recommendations. ${ }^{46}$ Meanwhile, the latter is characterized by difficulties in research accessibility, "unintelligibility of statistical analyses and results and its political implications" and the nature and relevance of the research evidence to the organization. ${ }^{39}$ Moore et al. suggest that in order to address these challenges, there must be: 1) available and efficient information system to ensure ready access to research findings and summaries; 2) an interactive environment that allows frequent interaction between policymakers, researchers, and research users; 3) organizational support to accept research and innovations, and 4) appropriate tools and facilities. ${ }^{45}$

Therefore, for PCHRD to improve health outcomes, proper strategies must be in place to ensure utilization of researches on health technology development. Such strategies may include regular monitoring and evaluation, having clear operational guidelines, and considering different institutional contexts. Furthermore, with health technology development as its institutional goal, PCHRD must implement a health technology assessment that will inform decision makers of the "possible impacts and consequences of a new technology or a significant change in an old technology." 44

\section{CONCLUSIONS AND RECOMMENDATIONS}

The NUHRA sets the health research priorities in the country to aid in the efficient and effective allocation of research and development resources towards addressing the health needs of the country. However, for a research agenda to achieve this objective, there is a need to develop strategies to strengthen the prioritization process and to coordinate regional and national efforts. This study suggests building capacity for researchers to produce quality proposals and studies by increasing training and providing more scholarships for researchers, especially for those based in the regions. In addition, strategies on agenda and research dissemination 
must be embedded in the formulation process to sustain demand for health research, as well as a regular monitoring and evaluation system for ensuring contextual relevance of priorities based on emerging or reemerging arguments and for research utilization.

Furthermore, this study expressed the need for an adequate representation of all stakeholders in the formulation of the NUHRA to "legitimize" the process and truly reflect the health needs of the country. Given the existing gaps and challenges in stakeholder involvement in the previous NUHRAs, while the completed research outputs are aligned to the priorities of the agenda (thus responsive to the NUHRAs), they are not necessarily responsive to the health needs of the Philippines.

Lastly, for PCHRD-funded health technology development researches to positively influence overall health outcomes, outputs must be materialized and be used. Following this, the core agency must implement a health technology assessment to guarantee safety and to warrant proper use of the technology developed.

\section{Acknowledgments}

We are grateful to Prof. Marilyn Ellorin-Crisostomo and Dr. Katherine Villegas-Reyes, of the University of the Philippines Manila, for their helpful inputs in the preparation of the manuscript.

\section{Statement of Authorship}

All authors approved the final version submitted.

\section{Author Disclosure}

All authors declared no conflict of interest.

\section{Funding Source}

This paper was funded by the Philippine Council for Health Research and Development.

\section{REFERENCES}

1. Ghaffar A, IJsselmuiden C, Zicker F. Changing Mindsets: Research capacity strengthening in low and middle-income countries. Geneva: Global Forum for Health Research; 2008.

2. Dye C, Boerma T, Evans D, et al. The World Health Report 2013. Research for Universal Health Coverage. World Health Organization; 2013.

3. Davey S, Louis C, Francisco A, Nchinda T. The 10/90 Report on Health Research 2000. The 10/90 Report on Health Research 2000. Geneva: Global Forum for Health Research; 2000.

4. Congress of the Philippines. The LawPhil Project [Internet]. [cited 2019 Apr]. Available from: https://www.lawphil.net/statutes/repacts/ ra2013/ra_10532_2013.html

5. Ramos-Jimenez P, Aragones D, Mendoza Y, Togonon L, Yubia M, Amita G. Assessment of the 2011-2016 National Unified Health Research Agenda (NUHRA) and the 2011-2015 Regional Unified Health Research Agenda (RUHRA). Manila: Rainier Contract Research Services, Inc., 2015.

6. Tarrant C, Angell E, Baker R, et al. Responsiveness of primary care services: development of a patient-report measure - qualitative study and initial quantitative pilot testing. Southampton (UK): NIHR
Journals Library [Internet]. 2014 Nov [cited 2019 Apr]. Available from: https://www.ncbi.nlm.nih.gov/books/NBK263694/

7. PCHRD.2006 Annual Report: Nurturing inclusive diversity for health and development Department of Science and Technology; 2006.

8. PCHRD. 2007 Annual Report: PCHRD: 25 Years of Making Lives Better for the Filipinos. Department of Science and Technology; 2007.

9. PCHRD. 2008 Annual Report: Making Health Research Work for Filipinos. Department of Science and Technology; 2008.

10. PCHRD. 2009 Annual Report: PCHRD @ 27: Inclusiveness @ PNHRS: Building Bridges to, and Engaging, Stakeholders. 2009 Annual Report: Department of Science and Technology; 2009.

11. PCHRD. 2010 Annual Report: Drawing in Young Researchers into the Regional Health Research Systems. Department of Science and Technology; 2010.

12. PCHRD. 2011 Annual Report: PCHRD @ 29: Challenges and Innovations in Local Health Technologies. Department of Science and Technology; 2011.

13. PCHRD. 2012 Annual Report: PCHRD 30 Health Research for Development. Department of Science and Technology; 2012.

14. PCHRD. 2013 Annual Report: People at the Center of Health and Health Research. Department of Science and Technology; 2013.

15. PCHRD. 2014 Annual Report: PCHRD: Going for convergence, across sectors, beyond national boundaries. Department of Science and Technology; 2014.

16. PCHRD. 2015 Annual Report: Leveling up, ready for the world. Department of Science and Technology; 2015.

17. PCHRD.2016 Annual Report: Ideas. Innovation. Impact. Department of Science and Technology; 2016.

18. PCHRD. 2017 Annual Report: Health Research and Innovation for the People. Department of Science and Technology; 2017.

19. NUHRA, 2006-2010. Manila: Philippine Council for Health Research and Development.

20. NUHRA, 2011-2016. Manila: Philippine Council for Health Research and Development.

21. DOH. Philippine Health Agenda 2016-2022: All for Health towards Health for All [Internet]. 2016 [cited 2019 Apr]. Available from: http://www.doh.gov.ph/sites/default/files/basic-page/Philippine Health Agenda_Dec1_1.pdf

22. Thomas DR. A general inductive approach for analyzing qualitative evaluation data. Am J Eval. 2006; 27(2):237-46.

23. Wright J, Williams R, Wilkinson JR. Development and importance of health needs assessment. BMJ. 1998; 316(7140):1310-3.

24. Vaughan JP, Morrow RH. Manual of epidemiology for district health management. New Delhi: CBS Publishers; 1994.

25. Edwards N, Kaseje D, Kahwa E. Building and evaluating research capacity in healthcare systems: case studies and innovative models. Lansdowne, Cape Town, South Africa: UCT Press; 2016.

26. Kabra R, Ali M, Gulmezoglu AM, Say L. Research capacity for sexual and reproductive health and rights. Bull World Health Organ. 2016; 94(7):549-50.

27. Bates I, Akoto AY, Ansong D, et al. Evaluating health research capacity building: an evidence-based tool. PLoS Med. 2006; 3(8):e299.

28. Whitworth JA, Kokwaro G, Kinyanjui S, et al. Strengthening capacity for health research in Africa. Lancet. 2008; 372(9649):1590-3.

29. Lansang MA, Dennis R. Building capacity in health research in the developing world. Bull World Health Organ. 2004; 82(10):764-70.

30. The Global Innovation Index (GII) 2013-2017 [Internet]. The Global Innovation Index (GII) 2013-2017 [cited 2019 Apr]. Available from: http://dost.gov.ph/phocadownload/Downloads/Statistics/Global_ Innovation_Index_2013-2017.pdf

31. Current Status on Science and Technology in ASEAN Countries [Internet]. Current Status on Science and Technology in ASEAN Countries. Japan Science and Technology Agency; 2015 [cited 2019 Apr]. Available from: https://www.jst.go.jp/crds/pdf/en/CRDSFY2014-OR-02_EN.pdf

32. Ramos-Jimenez P, Arguelles M. The Philippine National Health Research System: an assessment of the National Unified Health Research Agenda (2006-1010). Manila: Rainier Contract Research Services, Inc., 2010. 
33. Mahmood S, Hort K, Ahmed S, Salam M, Cravioto A. Strategies for capacity building for health research in Bangladesh: role of core funding and a common monitoring and evaluation framework. Health Res Policy Syst. 2011; 9:31.

34. Minja H, Nsanzabana C, Maure C, et al. Impact of Health Research Capacity Strengthening in Low- and Middle-Income Countries: The Case of WHO/TDR Programmes. PLoS Negl Trop Dis. 2011; 5(10):e1351.

35. Kirigia JM, Ota MO, Shongwe B. Swaziland National Health Research Agenda: the formulation process. Int Arch Med. 2017; 10.

36. COHRED. Health Research for Development: the continuing challenge [Internet]. Health Research for Development: the continuing challenge. 2000 [cited 2019 Apr]. Available from: http://www.cohred. org/downloads/727.pdf

37. PATH. An Advocacy Strategy for Adoption and Dissemination of the WHO Policy on TB Infection Control in Health-Care Facilities, Congregate Settings and Households [Internet]. An Advocacy Strategy for Adoption and Dissemination of the WHO Policy on TB Infection Control in Health-Care Facilities, Congregate Settings and Households. 2010 [cited 2019 Apr]. Available from: http:// www.tbonline.info/media/uploads/documents/advocacy_strategy_ for_adoption_and_dissemination_on_the_policy_on_tb_control_in_ health_care_facilities,_congregate_settings_and_households_(2010). pdf

38. Cole DC, Nyirenda LJ, Fazal N, Bates I. Implementing a national health research for development platform in a low-income country - a review of Malawi's Health Research Capacity Strengthening Initiative. Health Res Policy Syst. 2016; 14:24.
39. Lopez J, Dizon T. Towards a Relevant and Actionable Philippine Health Research Agenda: A Review. 2017. Unpublished

40. Lazarus J, France T. A new era for the WHO health system building blocks? [Internet]. Health Systems Global. [cited 2018 Apr]. Available from: http://www.healthsystemsglobal.org/blog/9/A-new-era-for-theWHO-health-system-building-blocks-.html

41. Yoshida S, Wazny K, Cousens S, Chan KY. Setting health research priorities using the CHNRI method: III. Involving stakeholders. J Glob Health. 2016; 6(1).

42. Tomlinson M, Chopra M, Hoosain N, Rudan I. A review of selected research priority setting processes at national level in low and middle income countries: towards fair and legitimate priority setting. Health Res Policy Syst. 2011; 9:19.

43. Population Council. Maximizing utilization of research. FRONTIERS Legacy Series. 2008.http://www.popcouncil.org/ uploads/pdfs/frontiers/legacy/08ResearchUtilization.pdf.

44. Goodman CS. HTA 101: Introduction to Health Technology Assessment. Bethesda, MD: National Library of Medicine (US); 2014.

45. Moore G, Todd A, Redman S. Strategies to increase the use of evidence from research in population health policy and programs: a rapid review. 2009.

46. Solidum G. Research Utilization among Nurse Supervisors in Two Local Government Hospitals in Manila, Philippines [Abstract]. IAMURE International Journal of Health Education. 2014; 6(1). 